\title{
From Tree Unitarity to Top Quark Physics in 5D Higgsless Models
}

\author{
Christian Schwinn ${ }^{* \dagger}$ \\ Institut für Physik, Johannes-Gutenberg-Universität \\ Staudingerweg 7, D-55099 Mainz, Germany \\ E-mail: schwinn@thep.physik.uni-mainz.de
}

In five dimensional models of Higgsless electroweak symmetry breaking, tree level unitarity in gauge boson scattering is restored by the exchange of gauge boson Kaluza-Klein modes instead of a Higgs boson. Unitarity of scattering amplitudes involving top quarks requires also the KaluzaKlein modes of the third family quarks. It is shown that the relevant unitarity cancellations are consistent with gauge symmetry breaking by boundary conditions. These results are used to constrain the couplings of the top quark to Kaluza-Klein modes and the implications for collider phenomenology are discussed.

International Europhysics Conference on High Energy Physics

July 21st - 27th 2005

Lisboa, Portugal

\footnotetext{
* Speaker.

†Work supported by by the Deutsche Forschungsgemeinschaft through the Graduiertenkolleg 'Eichtheorien' at Mainz University
} 


\section{Introduction: Tree unitarity in Higgsless models}

One of the motivations for expecting new physics at the $\mathrm{TeV}$ scale is the violation of perturbative unitarity-implying the onset of strong interactions-in the scattering of massive gauge bosons at the scale $\Lambda \sim 1-2 \mathrm{TeV}$ in a theory without a Higgs boson or other new particles. Tree level unitarity in $W^{+} W^{-} \rightarrow W^{+} W^{-}$scattering can be restored by introducing scalars $H$ or new vector bosons $V$ whose couplings satisfy the unitarity sum rule (SR)

$$
4 m_{W}^{2} g_{W W \gamma}^{2}+\left(4 m_{W}^{2}-3 m_{Z}^{2}\right) g_{W W Z}^{2}=\sum_{H} g_{W W H}^{2}+\sum_{V}\left(3 m_{V}^{2}-4 m_{W}^{2}\right) g_{W W V}^{2}
$$

The conventional solution to such SRs-realized in the Higgs mechanism-introduces one ore more scalar particles and no additional vector bosons. The alternative possibility of additional vector bosons and no scalar is realized in a Kaluza-Klein (KK) gauge theory and has led to the construction of higher dimensional electroweak Higgsless models [1]. With the lightest KK-modes at $500-700 \mathrm{GeV}$, these models remain weakly coupled up to a cutoff of 5-10 TeV implied by partial wave unitarity [2]. The gauge symmetry is broken at the boundaries of the extra dimension by assigning Dirichlet boundary conditions (BCs) to the broken gauge fields while the unbroken ones satisfy Neumann BCs. The consistency of these BCs with unitarity [1, 3] is discussed in section 2 . The collider signatures of such models have been constrained using the unitarity SRs [4], resulting in a bound $g_{Z W W^{(1)}} \lesssim \frac{g_{Z W W} m_{Z}^{2}}{\sqrt{3} m_{W^{(1)}} m_{W}}$ on the coupling of the first KK-mode of the $W$. In section 3 the implications of a similar analysis in the top sector [5] for collider phenomenology are discussed.

\section{Consistency of boundary conditions in $5 \mathrm{D}$ gauge theories}

Consistency of the Becchi-Rouet-Stora-Tuytin (BRST) symmetry can be used as a simple criterion for the compatibility of symmetry breaking by BCs with the unitarity SRs [3]. The KK wavefunctions of the $4 \mathrm{D}$ components of the gauge bosons $A_{\mu}^{a,(n)}(x)$ will be denoted by $f_{n}^{a}(y)$ and those of the fifth component $A_{5}^{a,(n)}(x)$ by $g_{n}^{a}(y)$ where one can impose the relation $\partial_{y} f_{n}^{a}=m_{A^{a,(n)}} g_{n}^{a}$. The KK-modes of $A_{5}$ play the role of Goldstone bosons with the BRST-transformation $\delta_{\mathrm{BRST}} A_{5}^{\alpha}=$ $m_{A^{\alpha}} c^{\alpha}+T_{\alpha \beta}^{\gamma} A_{5}^{\beta} c^{\gamma}$ where a multi-index $\alpha=(a, n)$ is used. The constants $T_{\alpha \beta}^{\gamma}$ also enter the Lagrangian:

$$
\mathscr{L}_{K K}=-g^{\alpha \beta \gamma} \partial_{\mu} A_{v}^{\alpha} A^{\beta, \mu} A^{\gamma, \nu}-\frac{1}{2} T_{\beta \gamma}^{\alpha} A^{\alpha, \mu} A_{5}^{\beta} \overleftrightarrow{\partial_{\mu}} A_{5}^{\gamma}+\ldots
$$

The coupling constants are given in terms of the structure constants $f^{a b c}$ and the KK-wavefunctions:

$$
g^{\alpha \beta \gamma}=f^{a b c} \int \mathrm{d} y f^{\alpha}(y) f^{\beta}(y) f^{\gamma}(y) \quad, \quad T_{\beta \gamma}^{\alpha}=f^{a b c} \int \mathrm{d} y f^{\alpha}(y) g^{\beta}(y) g^{\gamma}(y)
$$

Requiring nilpotency of the BRST transformation, i.e. $\delta_{\mathrm{BRST}}^{2} A_{5}^{\alpha}=0$, results in the conditions

$$
m_{A^{\beta}} T_{\alpha \beta}^{\gamma}-m_{A^{\gamma}} T_{\alpha \gamma}^{\beta}=m_{A^{\alpha}} g^{\alpha \beta \gamma} \quad, \quad T_{\alpha \beta}^{\gamma} T_{\beta \varepsilon}^{\delta}-T_{\alpha \beta}^{\delta} T_{\beta \varepsilon}^{\gamma}=g^{\beta \delta \gamma} T_{\alpha \varepsilon}^{\beta}
$$

where the transformation of the ghost fields $\delta_{\mathrm{BRST}} c^{\alpha}=\frac{1}{2} g^{\alpha \beta \gamma} c^{\beta} c^{\gamma}$ has been used in addition. Inserting the explicit expressions (2.2), the first condition in (2.3) involves the values of the KKwavefunctions on the boundaries, and thus gives a criterion for the consistency of BCs:

$$
0=f^{a b c} \int \mathrm{d} y \partial_{y}\left(g^{\alpha} f^{\beta} f^{\gamma}\right)=\left.f^{a b c}\left(g^{\alpha} f^{\beta} f^{\gamma}\right)\right|_{0} ^{\pi R}
$$


One can show [3] that (2.4) is satisfied for Neumann BCs $\left.\partial_{y} f^{\alpha}\right|_{0, \pi R}=0=\left.g^{\alpha}\right|_{0, \pi R}$ and for Dirichlet BCs $\left.f^{\alpha}\right|_{0, \pi R}=0=\left.\partial_{y} g^{\alpha}\right|_{0, \pi R}$ as used for symmetry breaking in 5D Higgsless models. Using the solution of the first condition, the second condition in (2.3) gives the unitarity SR for the cancellation of terms growing like $E^{2}$ and can be verified using the completeness relations of the KK-wavefunctions. Therefore the unitarity SRs are not spoiled by symmetry breaking by BCs.

\section{Unitarity sum rules for fermions and implications for top quark physics}

Fermion masses in 5D Higgsless models can be generated by gauge invariant brane localized mass and kinetic terms for bulk fermions [6] that respect the relevant unitarity SRs [7]. Electroweak precision data require to delocalize the zero modes of light fermions so they decouple from KK gauge bosons [8] and collider signatures are expected to be limited to the third family. Realistic values of the $t$ and $b$ quark masses require their first KK-modes to be heavier than those of the $W$ and $Z$ [8]. This has been achieved recently in theory space inspired models [9, 10]. Following [4], unitarity SRs can be used to constrain the interactions of the KK-modes with the top-and bottom quarks [5]. The SRs for the process $W^{+} W^{-} \rightarrow t \bar{t}$ result in the condition [5]

$$
\left(g_{W t b}^{L}\right)^{2}=\sum_{n}\left[2 \frac{m_{B^{(n)}}}{m_{t}} g_{W t B^{(n)}}^{R} g_{W t B^{(n)}}^{L}-\left(g_{W t B^{(n)}}^{L}\right)^{2}-\left(g_{W t B^{(n)}}^{R}\right)^{2}\right]
$$

that has been recently verified in a concrete model [10]. This condition shows that the KK-modes of the bottom quark are necessary for the unitarity cancellations. Taking also processes like $Z Z \rightarrow$ $t \bar{t}$ and $Z W^{+} \rightarrow t \bar{b}$ into account and truncating after the first KK-level, the unitarity SRs lead to the estimates [5] $g_{W t B^{(1)}} \approx \frac{g}{2} \sqrt{\frac{m_{t}}{m_{B^{(1)}}}}$ and $g_{t t Z^{(1)}} \approx \frac{\sqrt{3} g}{4} \frac{m_{t} m_{Z^{(1)}}}{m_{W} m_{B^{(1)}}}$. As seen in figure 1, this choice of couplings suppresses the $W^{+} W^{-} \rightarrow t \bar{t}$ cross section at high energies.

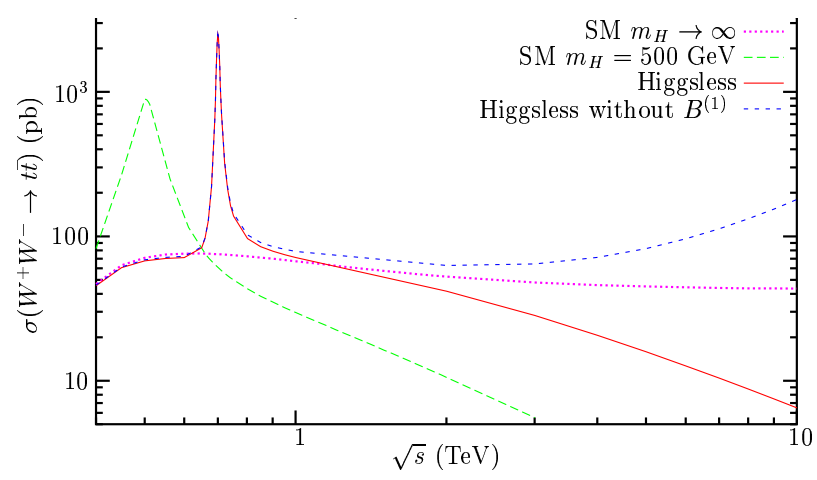

Figure 1: Cross section for $W^{+} W^{-} \rightarrow t \bar{t}$ in the SM with a Higgs resonance, the SM in the $m_{H} \rightarrow \infty$ limit, the Higgsless scenario (with $m_{Z}^{(1)}=700 \mathrm{GeV}$ and $m_{B^{(1)}}=2.5 \mathrm{TeV}$ ) and the Higgsless scenario without $B^{(1)}$

The above results can be used [5] to estimate the $Z^{(1)} \rightarrow t \bar{t}$ partial width to be about $14 \%$ of the $Z^{(1)} \rightarrow W^{+} W^{-}$partial width for $m_{B^{(1)}}=2.5 \mathrm{TeV}$, while it grows to $86 \%$ for $m_{B^{(1)}}=1 \mathrm{TeV}$. The most useful LHC signatures of a neutral gauge boson coupling mainly to third generation quarks are the associated production [11] in processes like $W b \rightarrow t Z^{(1)}$ or $g g \rightarrow t \bar{t} Z^{(1)}$. At an $e^{+} e^{-}$linear collider such a $Z$ resonance can be probed in the vector boson fusion process $W^{+} W^{-} \rightarrow \bar{t} t$ [12].

The properties of the $T^{(1)}$ can be compared to those of the heavy top in the littlest Higgs model [13]. The decay widths in the two scenarios are related by $\Gamma_{T^{(1)} \rightarrow t Z}^{\mathrm{HL}} \approx \frac{m_{T}}{4 m_{t}} \Gamma_{T}^{\mathrm{LH}}$ so in the 
Higgsless scenario the $T^{(1)}$ is broader, for instance $m_{T^{(1)}}=3 \mathrm{TeV}$ implies $\Gamma_{T^{(1)} \rightarrow t Z} \approx 270 \mathrm{GeV}$ compared to $\Gamma_{T}^{L H}=62 \mathrm{TeV}$ in the little Higgs model. The discovery reach of the LHC in $W-b$ fusion $q b \rightarrow q^{\prime} T$ has been estimated [13] as $m_{T}=2 \mathrm{TeV}$. The production cross section in the two scenarios can be related by $\sigma_{\mathrm{HL}}\left(W b \rightarrow T^{(1)}\right) \approx \frac{m_{b} m_{T}^{(1)}}{m_{t}^{2}} \sigma_{\mathrm{LH}}(W b \rightarrow T)$ so the detection of the $T^{(1)}$ in the Higgsless model is expected to be more challenging than in the Littlest Higgs model.

\section{Conclusions}

I have described theoretical methods to verify unitarity sum rules in higher dimensional Higgsless models and the phenomenological consequences in the top quark sector. As discussed in section 2, gauge symmetry breaking by boundary conditions used in these models is compatible with BRST symmetry, as required for a consistent quantization. Unitarity sum rules have been used in section 3 to constrain the couplings of the KK-modes of the third family quarks and gauge bosons to the top quark and the implications for collider phenomenology have been discussed.

\section{References}

[1] C. Csáki et al., Gauge theories on an interval: Unitarity without a Higgs, Phys. Rev. D69 (2004) 055006 [hep-ph/0305237].

[2] R. Chivukula, D. A. Dicus, and H.-J. He, Unitarity of compactified five dimensional Yang-Mills theory, Phys. Lett. B525 (2002) 175 [hep-ph/ 0111016 ]; M. Papucci, NDA and perturbativity in Higgsless models, hep-ph/0408058.

[3] T. Ohl and C. Schwinn, Unitarity, BRST symmetry and Ward identities in orbifold gauge theories, Phys. Rev. D70 (2004) 045019 [hep-ph / 0312263 ].

[4] A. Birkedal, K. Matchev, and M. Perelstein, Collider phenomenology of the Higgsless models, Phys. Rev. Lett. 94 (2005) 191803 [hep-ph / 0412278$].$

[5] C. Schwinn, Unitarity constraints on top quark signatures of Higgsless models, Phys. Rev. D71 (2005) 113005 [hep-ph/0504240].

[6] C. Csáki et al., Fermions on an interval: Quark and lepton masses without a Higgs, Phys. Rev. D70 (2004) 015012 [hep-ph/0310355].

[7] C. Schwinn, Higgsless fermion masses and unitarity, Phys. Rev. D69 (2004) 116005 [hep-ph/0402118].

[8] G. Cacciapaglia, C. Csáki, C. Grojean, and J. Terning, Curing the ills of Higgsless models: The S parameter and unitarity, Phys. Rev. D71 (2005) 035015 [hep-ph/ 0409126$].$

[9] H. Georgi, Chiral Fermion Delocalization in Deconstructed Higgsless Theories, hep-ph / 0508014.

[10] R. Foadi and C. Schmidt, An effective Higgsless theory: Satisfying electroweak constraints and a heavy top quark, hep-ph / 0509071.

[11] T. Han, G. Valencia, and Y. Wang, Hadron collider signatures for new interactions of top and bottom quarks, Phys. Rev. D70 (2004) 034002 [hep-ph/ 0405055 ].

[12] T. Han, Y. J. Kim, A. Likhoded, and G. Valencia, Top-quark couplings to TeV resonances at future lepton colliders, Nucl. Phys. B593 (2001) 415 [hep-ph / 0005306$].$

[13] T. Han, H. E. Logan, B. McElrath, and L.-T. Wang, Phenomenology of the little Higgs model., Phys. Rev. D67 (2003) 095004 [hep-ph/ 0301040$].$ 